\title{
Cyclophilin A-EMMPRIN interaction induces invasion of head and neck squamous cell carcinoma
}

\author{
MASAFUMI TAKAHASHI, SHINSUKE SUZUKI and KAZUO ISHIKAWA \\ Department of Otorhinolaryngology and Head and Neck Surgery, Akita University \\ Graduate School of Medicine, Akita 010-8543, Japan
}

Received July 11, 2011; Accepted August 10, 2011

DOI: $10.3892 /$ or.2011.1474

\begin{abstract}
Extracellular matrix remodeling crucial to tumorigenesis involves proteolytic enzymes, primarily matrix metalloproteinases (MMPs). MMP production is stimulated by multiple factors, including the extracellular matrix metalloproteinase inducer EMMPRIN/CD147. Overexpression of EMMPRIN, a member of the immunoglobulin superfamily, promotes invasion, metastasis, growth and survival of malignant cells. Cyclophilin A (CypA) is a multifunctional protein that promotes cancer progression in various cancer types. CypA can interact with and activate EMMPRIN; however, the role of CypA-EMMPRIN interaction in oncogenicity is not completely understood. To investigate tumorigenicity induced by the CypA-EMMPRIN interaction, we stimulated EMMPRINexpressing head and neck squamous cell carcinoma (HNSCC) cells with CypA. The 3-(4,5-dimethylthiazol-2-yl)-2,5-diphenyltetrazolium bromide dye assay revealed that HNSCC cell proliferation increased upon stimulation of the cells with CypA, whereas cisplatin-induced cell death decreased after stimulation. Gelatin zymography showed that CypA also induced MMP-9 up-regulation. Moreover, HNSCC cell invasion through Matrigel ${ }^{\mathrm{TM}}$-coated membranes was increased upon stimulation of cells with CypA. This elevated invasive potential was abrogated by an EMMPRIN function-blocking antibody. These findings suggest that CypA, through its interaction with EMMPRIN, contributes to HNSCC tumorigenesis.
\end{abstract}

\section{Introduction}

Matrix metalloproteinases (MMPs) are a subfamily of endopeptidases, enzymes that degrade components of the extracellular matrix (ECM). Because disruption of ECM, including the basement membrane, which serves as a barrier between tissue compartments, is a crucial step in tumor

Correspondence to: Dr Shinsuke Suzuki, Department of Otorhinolaryngology and Head and Neck Surgery, Akita University Graduate School of Medicine, Akita 010-8543, Japan

E-mail: suzukis@med.akita-u.ac.jp

Key words: cyclophilin A, EMMPRIN, head and neck squamous cell carcinoma progression, the role of MMPs has been studied in malignant tumors $(1,2)$. In these tumors, MMP overexpression correlates with increased cancer invasiveness, both in vitro and in vivo and results in a poor prognosis (3).

A growing number of reports suggest that MMP expression in cancer tissue is promoted through soluble factors, such as cytokines. In addition to several humoral factors, cell-cell interaction mediated by cell adhesion molecules such as the extracellular matrix metalloproteinase inducer EMMPRIN (also known as CD147) is important for MMP production in tumors (4-7).

EMMPRIN, a membrane glycoprotein greatly enriched on the surface of tumor cells (8-10), is known to stimulate increased MMP synthesis in tumor and neighboring stromal cells and promote tumor growth and lymphatic metastasis $(11,12)$. EMMPRIN is highly expressed in head and neck squamous cell carcinoma (HNSCC) cells (13). We previously showed that EMMPRIN plays an important role in HNSCC progression through MMP production and activation $(14,15)$.

Although increasing evidence implicates EMMPRIN in cancer progression, the precise mechanisms of EMMPRIN activation remain unclear. Homophilic EMMPRINEMMPRIN interaction has been proposed to be a mechanism by which EMMPRIN could induce MMP production $(7,16)$. In addition to the homophilic binding of EMMPRIN, recent studies reported that cyclophilin A (CypA) acts as a ligand for EMMPRIN $(17,18)$. The importance of the CypA-EMMPRIN interaction has been reported in rheumatoid arthritis (19), foam cell formation (17) and also in several solid tumors (20-22). However, the role of CypA and its interaction with EMMPRIN in HNSCC has not yet been evaluated.

In this study, we sought to investigate the functional role of CypA and its receptor EMMPRIN during the process of HNSCC progression in vitro. Using two HNSCC cell lines, we examined whether CypA induces tumorigenic behavior in HNSCC cells, such as cell proliferation, drug resistance and MMP expression. In addition, we evaluated CypA-induced HNSCC cell invasion, both with and without an EMMPRIN function-blocking antibody, to confirm the role of the CypAEMMPRIN interaction in HNSCC progression.

\section{Materials and methods}

Cell lines and culture. FaDu, a human hypopharyngeal squamous cell carcinoma cell line, was a kind gift from the 
Department of Cell Biology and Morphology, Akita University Graduate School of Medicine (Akita, Japan). SAS, a human tongue squamous cell carcinoma cell line, was purchased from the Cell Engineering Division of RIKEN BioResorce Center (Tsukuba, Japan). The cells were maintained in Dulbecco's modified Eagle's medium (DMEM; Gibco-Invitrogen, Grand Island, NY, USA) supplemented with $10 \%$ fetal bovine serum (FBS) in a humidified atmosphere containing $5 \% \mathrm{CO}_{2}$ at $37^{\circ} \mathrm{C}$. For stimulation experiments, the FaDu and SAS cells were preincubated with serum-free DMEM and subsequently incubated with serum-free medium containing $400 \mathrm{ng} / \mathrm{ml}$ of CypA (Sigma-Aldrich, St. Louis, MO, USA).

Antibodies. A goat anti-EMMPRIN antibody, H-200 (Santa Cruz Biotechnology Inc., Santa Cruz, CA, USA), was used for immunoblotting. A mouse anti-EMMPRIN function-blocking antibody, UM-8D6 (Research Diagnostics Inc., Flanders, NJ, USA), was used for the inhibition studies; its blocking activity has been described previously $(12,23)$.

Immunoblotting. The FaDu and SAS cells were lysed in detergent containing $1 \% \mathrm{NP}-40,150 \mathrm{mmol} / 1 \mathrm{NaCl}, 1 \mathrm{mmol} / \mathrm{l}$ EDTA, $0.1 \mathrm{mmol} / 1$ phenylmethylsulfonyl fluoride, $1 \mu \mathrm{g} / \mathrm{ml}$ leupeptin and $1 \mu \mathrm{g} / \mathrm{ml}$ aprotinin. Protein levels were then determined by the Bio-Rad protein assay method (Bio-Rad Laboratories). Total protein $(40 \mu \mathrm{g})$ was separated on $8 \%$ SDS-PAGE gels and transferred to nitrocellulose membranes using a semi-dry transfer machine (Bio-Rad Laboratories). The membranes were blocked with 5\% skim milk/TBS with Tween-20 (TBS-T) for $1 \mathrm{~h}$ at room temperature and then incubated overnight at $4^{\circ} \mathrm{C}$ with primary antibodies in 5\% skim milk in TBS-T. After washing thrice with TBS-T, the membranes were incubated for $1 \mathrm{~h}$ with a horseradish peroxidase-conjugated secondary antibody (Bio-Rad Laboratories) in 5\% skim milk in TBS-T. The filters were rinsed thrice with TBS-T, and the blots were developed using luminol (Santa Cruz Biotechnology Inc.) and visualized by autoradiography.

Proliferation assay. The FaDu and SAS cells were plated in triplicate at a density of $2 \times 10^{4}$ per well and allowed to seed overnight in a 96-well plate. The cells were grown in serum-free DMEM for $24 \mathrm{~h}$ prior to experimental treatments and then treated with the control vehicle PBS or CypA (400 ng/ml) in serum-free DMEM for $24 \mathrm{~h}$. Metabolically active cells were labeled with 3-(4,5-dimethylthiazol-2-yl)-2,5-diphenyltetrazoliumbromide (MTT) tetrazolium for $1 \mathrm{~h}$ and measured at $570 \mathrm{~nm}$.

Drug resistance assay. Drug resistance was determined by the MTT (Sigma-Aldrich) assay. Briefly, the FaDu and SAS cells were plated in triplicate at a density of $2 \times 10^{4}$ per well and allowed to seed overnight in a 96-well plate. The cells were grown in serum-free DMEM for $24 \mathrm{~h}$ prior to experimental treatments and then treated with either the control vehicle DMSO or cisplatin $(0.5 \mu \mathrm{M})$ with or without CypA $(400 \mathrm{ng} / \mathrm{ml})$ in DMEM with $10 \%$ FBS for $24 \mathrm{~h}$. Metabolically active cells were labeled with MTT tetrazolium for $1 \mathrm{~h}$ and measured at $570 \mathrm{~nm}$.

Gelatin zymography. The FaDu and SAS cells were cultured overnight using ordinary serum-containing DMEM, washed with serum-free DMEM, and cultured further for $48 \mathrm{~h}$ in

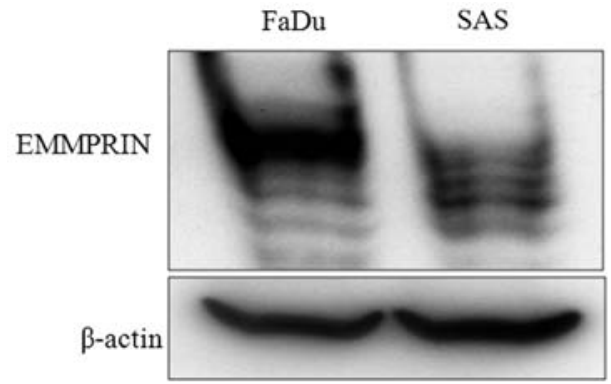

Figure 1. EMMPRIN expression in HNSCC cells. EMMPRIN protein expression was determined by immunoblotting in the HNSCC cell lines FaDu and SAS. Both HNSCC cell lines showed high EMMPRIN protein expression.

serum-free DMEM with or without 400 ng/ml CypA. This was followed by detection of gelatinolytic activity in the conditioned media by gelatin zymography. Using a 7.5\% separating gel containing $0.3 \mathrm{mg} / \mathrm{ml}$ gelatin, the conditioned medium was resolved by SDS-PAGE under non-reducing conditions. The gels were washed twice in $2.5 \%$ (w/v) Triton X-100 for $30 \mathrm{~min}$ at room temperature to remove SDS and then incubated for $24 \mathrm{~h}$ at $37^{\circ} \mathrm{C}$ in reaction buffer containing $50 \mathrm{mM}$ Tris- $\mathrm{HCl}$ (pH 7.6), $5 \mathrm{mM} \mathrm{CaCl}_{2}$ and $2.5 \%$ Triton X-100. Subsequently, the gels were stained with $2.5 \%(\mathrm{w} / \mathrm{v})$ Coomassie Brilliant Blue R-250 in $30 \%(\mathrm{v} / \mathrm{v})$ methanol and $10 \%(\mathrm{v} / \mathrm{v})$ acetic acid. After destaining with $30 \%$ methanol and $10 \%$ acetic acid, gelatinolytic activities on the gel were detected as clear bands on a blue background of undigested gelatin.

Matrigel invasion assay. Cell invasiveness was evaluated in vitro using Matrigel ${ }^{\mathrm{TM}}$-coated semipermeable, modified Boyden chamber inserts with a pore size of $8 \mu \mathrm{m}$ (BectonDickinson/Biocoat). The FaDu and SAS cells $\left(2.5 \times 10^{4}\right)$ were plated in the insert containing serum-free medium with or without $10 \mu \mathrm{g} / \mathrm{ml}$ of the EMMPRIN function-blocking antibody. The lower chamber contained DMEM plus $10 \%$ FBS with or without $400 \mathrm{ng} / \mathrm{ml}$ of CypA, which served as a chemoattractant. To control the effect of inhibitors on cell growth, the cells were also plated in parallel in a 96-well plate under identical conditions. After $48 \mathrm{~h}$ of treatment at $37^{\circ} \mathrm{C}$ in a $5 \% \mathrm{CO}_{2}$ incubator, the cells in the insert were removed by wiping gently with a cotton swab. Cells on the reverse side of the insert were fixed and stained with Diff-Quik ${ }^{\circledR}$ (Sysmex, Kobe, Japan), according to the manufacturer's instructions. Invading cells in four representative fields were counted by light microscopy at x200 magnification. Mean \pm SE was calculated from three independent experiments. Metabolically active cells were identified by the MTT assay. The number of invading cells was adjusted accordingly.

Statistical analysis. The Wilcoxon-Mann-Whitney two-tailed exact test was used to assess the statistical significance of differences in proliferation, drug resistance, MMP-9 expression and invasion studies.

\section{Results}

EMMPRIN expression in HNSCC cell lines. To evaluate the role of the interaction between EMMRPIN and its ligand CypA, 


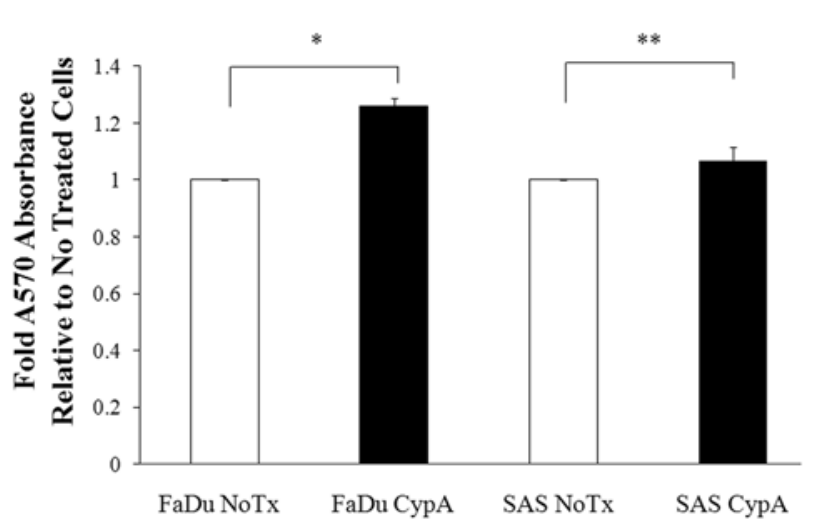

Figure 2. CypA increases HNSCC proliferation. The FaDu and SAS cells were plated and allowed to seed overnight in a 96-well plate. The cells were grown in serum-free DMEM for $24 \mathrm{~h}$ prior to experimental treatments and then treated with the control vehicle PBS (NoTx) or $400 \mathrm{ng} / \mathrm{ml}$ of CypA (CypA) in serum-free DMEM for $24 \mathrm{~h}$. Metabolically active cells were labeled with MTT tetrazolium for $1 \mathrm{~h}$ and measured at $570 \mathrm{~nm}$. Mean $\pm \mathrm{SE}$ was calculated from three independent experiments. CypA increased proliferation of $\mathrm{FaDu}\left({ }^{*} \mathrm{P}=0.036\right)$ and $\mathrm{SAS}$ cells $\left({ }^{* *} \mathrm{P}=0.03\right)$.

EMMPRIN protein expression was determined by immunoblotting in the HNSCC cell lines FaDu and SAS. Both HNSCC cell types showed high EMMPRIN protein expression (Fig. 1).

CypA increases HNSCC proliferation and drug resistance. Tumorigenetic steps, such as proliferation, anti-apoptosis and cell migration, are crucial for malignant progression. To assess the contribution of CypA to HNSCC tumorigenicity, the FaDu and SAS cells were cultured with $400 \mathrm{ng} / \mathrm{ml} \mathrm{CypA}$ and then a cell proliferation assay was performed. In both HNSCC cell lines, cell proliferation was significantly increased with CypA than without (Fig. 2).

Chemotherapy is the standard approach for treatment of HNSCC patients. The benefits of cisplatin were first shown 20 years ago, and it remains a commonly used agent, either alone or in combination with other chemotherapeutic drugs. To investigate the role of CypA in response to cisplatin, the $\mathrm{FaDu}$ and SAS cells were treated with cisplatin with or without CypA and then the MTT assay was performed. The FaDu and SAS cells cultured with CypA showed higher metabolic

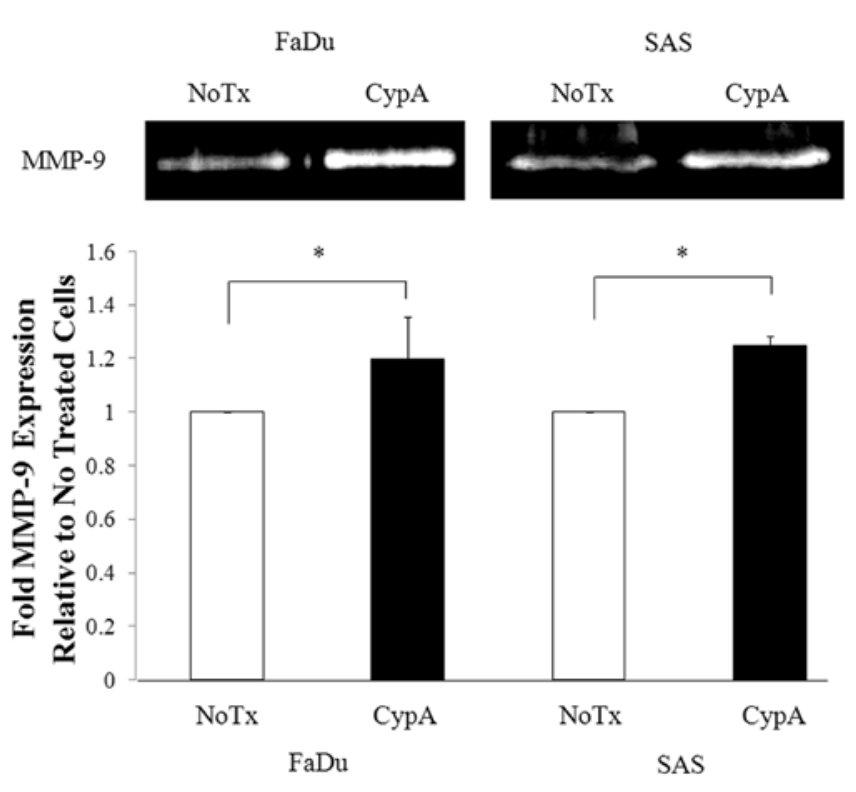

Figure 4. CypA enhances MMP-9 expression in HNSCC. The FaDu and SAS cells were cultured overnight using ordinary serum-containing DMEM, washed with serum-free DMEM and cultured further for $48 \mathrm{~h}$ using serumfree DMEM with or without $400 \mathrm{ng} / \mathrm{ml}$ CypA. This was followed by detection of gelatinolytic activity in the conditioned media by gelatin zymography. A representative result of Gelatin zymography (top) and the relative MMP-9 expression on stimulation with CypA (CypA) compared to control (NoTx). Mean \pm SE was calculated from four independent experiments. Gelatin zymography detected up-regulated expression of MMP-9, not MMP-2, in HNSCC cells ( $\mathrm{P}=0.0369)$.

activity with cisplatin treatment (Fig. 3). These results suggest that CypA contributes to HNSCC tumorigenicity.

MMP-9 expression is induced on stimulation of cells with CypA. MMPs contribute to tumor progression. In particular, the gelatinases MMP-2 and MMP-9 are known to be important in HNSCC progression. Therefore, we assessed whether CypA participates in MMP expression by gelatin zymography. MMP-9 expression increased on stimulating the cells with CypA (Fig. 4). However, MMP-2 expression was not observed in the FaDu or SAS cells, even after stimulation.
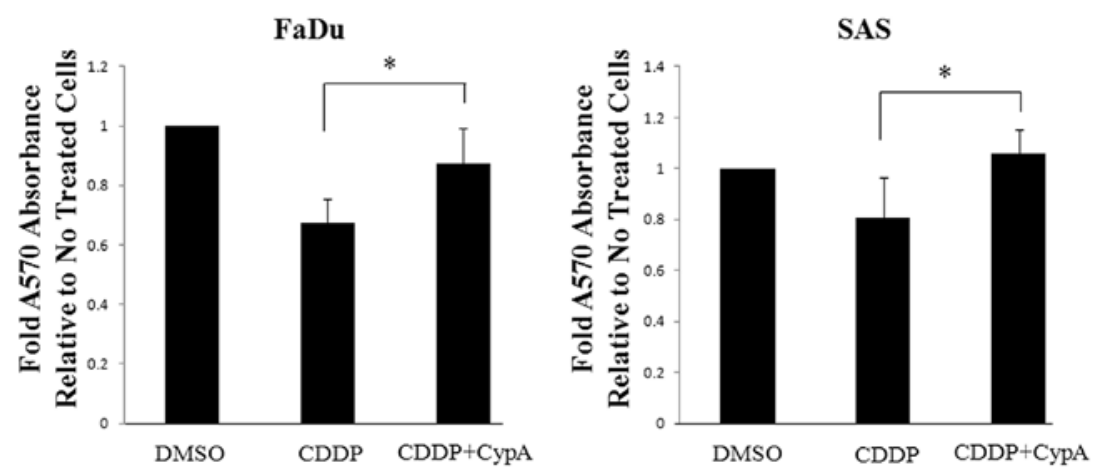

Figure 3. CyPA induces HNSCC drug resistance. Drug resistance was determined by the MTT assay. The cells were grown in serum-free DMEM for $24 \mathrm{~h}$ prior to experimental treatments and then treated with the control vehicle DMSO (NoTx) or $0.5 \mu \mathrm{M}$ of cispalin (CDDP) or cisplatin with $400 \mathrm{ng} / \mathrm{ml}$ of CypA $(\mathrm{CDDP}+\mathrm{CypA})$ in DMEM with 10\% FBS for $24 \mathrm{~h}$. Metabolically active cells were labeled with MTT tetrazolium for $1 \mathrm{~h}$ and measured at $570 \mathrm{~nm}$. Mean \pm SE was calculated from five independent experiments. The MTT assay revealed that HNSCC cell activity was reduced by cisplatin but this reduction was recovered on stimulation with CypA (" $\mathrm{P}=0.0495)$. 


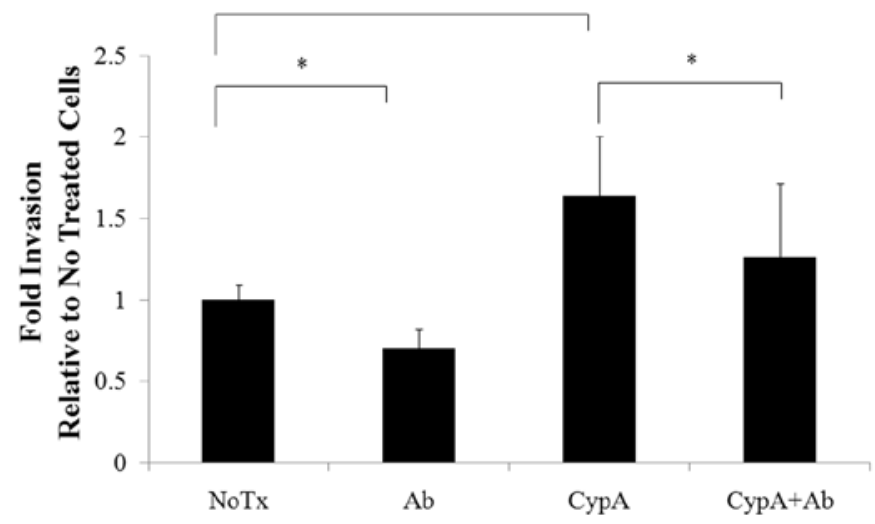

Figure 5. CypA induces HNSCC cell invasion through EMMPRIN. Invasion assay was performed to evaluate cell invasiveness in vitro. The FaDu and SAS cells were plated in the insert containing serum-free medium with or without $10 \mu \mathrm{g} / \mathrm{ml}$ of the EMMPRIN function-blocking antibody. The lower chamber contained DMEM plus 10\% FBS with or without $400 \mathrm{ng} / \mathrm{ml} \mathrm{CypA}$ which served as a chemoattractant. After $48 \mathrm{~h}$ of treatment, invading cells in four representative fields were counted by light microscopy at x200 magnification. The number of invading cells was adjusted accordingly. Mean \pm SE was calculated from six independent experiments. The number of invading cells increased on stimulation with CypA (CypA) compared to no treated cells (NoTx) and this increment was inhibited by the EMMPRIN functionblocking antibody $(\mathrm{CypA}+\mathrm{Ab})\left({ }^{*} \mathrm{P}<0.05\right)$.

CypA induces HNSCC cell invasion through EMMPRIN. Because tumor cell invasion is one of the most lethal steps for cancer progression, controlling tumor invasion has been focused on, in the study of tumor suppression. To understand the mechanism of HNSCC cell invasion, the SAS cells were plated on a Matrigel ${ }^{\mathrm{TM}}$ invasion chamber and stimulated with CypA. The results of this invasion assay are shown in Fig. 5. The invasiveness of both HNSCC cell lines was up-regulated when the cells were cocultured with CypA. To determine whether this increased invasiveness was mediated by the CypA-EMMPRIN interaction, an EMMPRIN function-blocking antibody was added to the invasion system. Hence, the increased invasiveness was efficiently reduced by the antibody. This result indicates that CypA induces HNSCC cell invasion through an interaction with EMMPRIN.

\section{Discussion}

In this study, we showed CypA induces cell proliferation, drug resistance, MMP-9 expression and invasion of two HNSCC cell lines expressing EMMPRIN. Notably, functional blocking of EMMPRIN abolished an increase in CypA-induced HNSCC cell invasiveness. These results suggest that CypA promotes HNSCC tumor progression and that EMMPRIN is the key mediator for these tumor phenotypes.

The importance of EMMPRIN in the steps of cancer progression such as tumor growth and lymphatic metastasis is widely known based on recent studies including HNSCC $(12-15,24,25)$. Several studies reported that EMMPRIN blocking or silencing is efficient for cancer suppression (26-30). Hence, a more detailed understanding of the mechanisms underlying EMMRPIN-mediated cancer progression, which could contribute to improvement in HNSCC prognosis, is necessary.

We previously reported that EMMPRIN plays an important role in MMP-2 production and activation and also in migration of HNSCC cells through a homophilic EMMPRIN-EMMPRIN interaction. In addition to this homophilic interaction, CypA has been described as a ligand for EMMPRIN that binds to EMMPRIN and transmits a signal to downstream cascades (18).

CypA, a ubiquitously distributed intracellular protein belonging to the immunophilin family (31), is a target of the immunosuppressive drug cyclosporine A. CypA is secreted from cells in response to inflammatory stimulation and is a multifunctional cytokine involved in many biological processes, such as protein folding, cellular signaling, immunosuppression, and apoptosis, in many cell types. Reflecting these biological potentials, the role of CypA has been studied in many types of malignant tumors, such as non-small cell lung carcinoma, pancreatic cancer, bladder cancer, hepatocellular carcinoma, sarcoma and breast cancer (20-22). These studies showed that CypA expression is up-regulated in tumor tissues and that CypA induces cancer cell proliferation and MMP expression, resulting in a poor prognosis. Our results show the oncogenic role of CypA in HNSCC, and these findings contribute to our understanding of the mechanisms underlying HNSCC progression.

Of all the MMPs, we focused on gelatinase expression because these enzymes are indispensable for basement membrane degradation, which is crucial not only for local cancer invasion but also for metastasis $(2,32)$. In this study, we found that MMP-9, not MMP-2, was induced on stimulation of cells with CypA. We could not detect MMP-2 expression, which is perplexing because MMP-2 is an equally important enzyme for degradation of the basement membrane typical in invasion and metastasis. Conventionally, tumor cells were considered the major source of MMPs in cancer tissue. However, current studies indicate that most MMPs in cancer tissue, including MMP-2 and MMP-9, are produced by stromal cells, such as fibroblasts, through cell surface EMMPRIN $(11,12)$. We previously showed that laryngeal cancer cells stimulate MMP-2 expression and induce its activator, MT-1MMP, mainly in fibroblasts (14). CypA up-regulates MMP-9 expression in some non-cancer inflammatory situations $(17,19)$. Our results show that CypA can induce MMP-9 production in cancer cells. However other MMPs, especially the gelatinase MMP-2, are also important for cancer progression. A more detailed study of CypA-induced MMP expression in tumor tissues will be necessary in future.

Studies of the CypA-EMMPRIN interaction in cancer are currently in progress. The potential role of the CypAEMMPRIN interaction in cell activation has been investigated in cancer (20) and some immunological diseases $(18,33)$. Although accumulating evidence supports the importance of the CypA-EMMPRIN interaction, there is a paucity of data in case of HNSCC progression. Cancer cell invasion is the most crucial step in cancer progression, which correlates with metastasis and directly relates to poor prognosis in HNSCC patients (34). Therefore, a detailed understanding of the invasion mechanism is critical for cancer prevention, especially HNSCC. Our invasion study shows that CypA 
induces HNSCC cell invasion and that this invasiveness is mediated by EMMPRIN. This finding may contribute to the development of effective therapeutic strategies for HNSCC treatment.

Indeed, anti-EMMPRIN therapy has been preclinically investigated in colon cancer (27) and HNSCC (26). These studies show great potential in not only in vitro but also ex vivo models. Unfortunately, HNSCC treatment outcomes have not improved for the last three decades (35). Agents such as cisplatin are among the most potent agents available for HNSCC treatment; however, additional, novel strategies appear necessary to improve HNSCC prognosis. EGFR is a cell surface protein that is overexpressed in cancer cells, and EGFR expression is relatively high in HNSCC cells (36). Cancer-specific molecular targeting therapy, such as the EGFR-targeting drug cetuximab, shows great potential in HNSCC treatment (37). Similarly to EGFR, EMMPRIN is specifically overexpressed in various cancer types, including HNSCC (13) and we propose that EMMPRIN is an ideal new target for HNSCC therapy.

\section{Acknowledgements}

This study was supported by a Grant-in-Aid for Young Scientists (B) 21791590 .

\section{References}

1. Barsky SH, Siegal GP, Jannotta F and Liotta LA: Loss of basement membrane components by invasive tumors but not by their benign counterparts. Lab Invest 49: 140-147, 1983.

2. Liotta LA, Tryggvason K, Garbisa S, Hart I, Foltz CM and Shafie S: Metastatic potential correlates with enzymatic degradation of basement membrane collagen. Nature 284: 67-68, 1980 .

3. Nelson AR, Fingleton B, Rothenberg ML and Matrisian LM: Matrix metalloproteinases: biologic activity and clinical implications. J Clin Oncol 18: 1135-1149, 2000

4. Biswas $C$ and Nugent MA: Membrane association of collagenase stimulatory factor(s) from B-16 melanoma cells. J Cell Biochem 35: 247-258, 1987.

5. Sameshima T, Nabeshima K, Toole BP, et al: Glioma cell extracellular matrix metalloproteinase inducer (EMMPRIN) (CD147) stimulates production of membrane-type matrix metalloproteinases and activated gelatinase $\mathrm{A}$ in co-cultures with brain-derived fibroblasts. Cancer Lett 157: 177-184, 2000.

6. Guo H, Zucker S, Gordon MK, Toole BP and Biswas C: Stimulation of matrix metalloproteinase production by recombinant extracellular matrix metalloproteinase inducer from transfected Chinese hamster ovary cells. J Biol Chem 272: 24-27, 1997.

7. Li R, Huang L, Guo H and Toole BP: Basigin (murine EMMPRIN) stimulates matrix metalloproteinase production by fibroblasts. J Cell Physiol 186: 371-379, 2001.

8. Caudroy S, Polette M, Tournier JM, et al: Expression of the extracellular matrix metalloproteinase inducer (EMMPRIN) and the matrix metalloproteinase-2 in bronchopulmonary and breast lesions. J Histochem Cytochem 47: 1575-1580, 1999.

9. Polette M, Gilles C, Marchand V, et al: Tumor collagenase stimulatory factor (TCSF) expression and localization in human lung and breast cancers. J Histochem Cytochem 45: 703-709, 1997.

10. Muraoka K, Nabeshima K, Murayama T, Biswas C and Koono M: Enhanced expression of a tumor-cell-derived collagenase-stimulatory factor in urothelial carcinoma: its usefulness as a tumor marker for bladder cancers. Int J Cancer 55: 19-26, 1993.

11. Kanekura T, Chen X and Kanzaki T: Basigin (CD147) is expressed on melanoma cells and induces tumor cell invasion by stimulating production of matrix metalloproteinases by fibroblasts. Int J Cancer 99: 520-528, 2002.
12. Bordador LC, Li X, Toole B, et al: Expression of emmprin by oral squamous cell carcinoma. Int J Cancer 85: 347-352, 2000.

13. Rosenthal EL, Shreenivas S, Peters GE, Grizzle WE, Desmond R and Gladson CL: Expression of extracellular matrix metalloprotease inducer in laryngeal squamous cell carcinoma. Laryngoscope 113: 1406-1410, 2003.

14. Suzuki S, Sato M, Senoo H and Ishikawa K: Direct cell-cell interaction enhances pro-MMP-2 production and activation in co-culture of laryngeal cancer cells and fibroblasts: involvement of EMMPRIN and MT1-MMP. Exp Cell Res 293: 259-266, 2004.

15. Hanata K, Yamaguchi N, Yoshikawa K, et al: Soluble EMMPRIN (extra-cellular matrix metalloproteinase inducer) stimulates the migration of HEp-2 human laryngeal carcinoma cells, accompanied by increased MMP-2 production in fibroblasts. Arch Histol Cytol 70: 267-277, 2007.

16. Sun J and Hemler ME: Regulation of MMP-1 and MMP-2 production through CD147/extracellular matrix metalloproteinase inducer interactions. Cancer Res 61: 2276-2281, 2001.

17. Seizer P, Schonberger T, Schott M, et al: EMMPRIN and its ligand cyclophilin A regulate MT1-MMP, MMP-9 and M-CSF during foam cell formation. Atherosclerosis 209: 51-57, 2010.

18. Yurchenko V, Constant S and Bukrinsky M: Dealing with the family: CD147 interactions with cyclophilins. Immunology 117: 301-309, 2006.

19. Yang Y, Lu N, Zhou J, Chen ZN and Zhu P: Cyclophilin A up-regulates MMP-9 expression and adhesion of monocytes/ macrophages via CD147 signalling pathway in rheumatoid arthritis. Rheumatology (Oxford) 47: 1299-1310, 2008.

20. Li M, Zhai Q, Bharadwaj U, et al: Cyclophilin A is overexpressed in human pancreatic cancer cells and stimulates cell proliferation through CD147. Cancer 106: 2284-2294, 2006.

21. Campa MJ, Wang MZ, Howard B, Fitzgerald MC and Patz EF Jr: Protein expression profiling identifies macrophage migration inhibitory factor and cyclophilin a as potential molecular targets in non-small cell lung cancer. Cancer Res 63: 1652-1656, 2003.

22. Meza-Zepeda LA, Forus A, Lygren B, et al: Positional cloning identifies a novel cyclophilin as a candidate amplified oncogene in 1q21. Oncogene 21: 2261-2269, 2002.

23. Koga K, Nabeshima K, Aoki M, et al: Emmprin in epithelioid sarcoma: expression in tumor cell membrane and stimulation of MMP-2 production in tumor-associated fibroblasts. Int J Cancer 120: 761-768, 2007.

24. Biswas C, Zhang Y, DeCastro R, et al: The human tumor cellderived collagenase stimulatory factor (renamed EMMPRIN) is a member of the immunoglobulin superfamily. Cancer Res 55: 434-439, 1995.

25. Riethdorf S, Reimers N, Assmann V, et al: High incidence of EMMPRIN expression in human tumors. Int J Cancer 119: 1800-1810, 2006.

26. Dean NR, Newman JR, Helman EE, et al: Anti-EMMPRIN monoclonal antibody as a novel agent for therapy of head and neck cancer. Clin Cancer Res 15: 4058-4065, 2009.

27. Baba M, Inoue M, Itoh K and Nishizawa Y: Blocking CD147 induces cell death in cancer cells through impairment of glycolytic energy metabolism. Biochem Biophys Res Commun 374: $111-116,2008$.

28. Su J, Chen X and Kanekura T: A CD147-targeting siRNA inhibits the proliferation, invasiveness, and VEGF production of human malignant melanoma cells by down-regulating glycolysis. Cancer Lett 273: 140-147, 2009.

29. Schneiderhan W, Scheler M, Holzmann KH, et al: CD147 silencing inhibits lactate transport and reduces malignant potential of pancreatic cancer cells in in vivo and in vitro models. Gut 58: 1391-1398, 2009.

30. Zhu C, Pan Y, He B, et al: Inhibition of CD147 gene expression via RNA interference reduces tumor cell invasion, tumorigenicity and increases chemosensitivity to cisplatin in laryngeal carcinoma Hep2 cells. Oncol Rep 25: 425-432, 2010.

31. Galat A: Peptidylproline cis-trans-isomerases: immunophilins. Eur J Biochem 216: 689-707, 1993.

32. Matrisian LM and Bowden GT: Stromelysin/transin and tumor progression. Semin Cancer Biol 1: 107-115, 1990.

33. Pushkarsky T, Yurchenko V, Vanpouille C, et al: Cell surface expression of CD147/EMMPRIN is regulated by cyclophilin 60 . J Biol Chem 280: 27866-27871, 2005. 
34. Johnson JT, Myers EN, Bedetti CD, Barnes EL, Schramm VL Jr and Thearle PB: Cervical lymph node metastases. Incidence and implications of extracapsular carcinoma. Arch Otolaryngol 111: 534-537, 1985.

35. Jemal A, Siegel R, Ward E, Murray T, Xu J and Thun MJ: Cancer statistics, 2007. CA Cancer J Clin 57: 43-66, 2007.

36. Grandis JR and Tweardy DJ: Elevated levels of transforming growth factor alpha and epidermal growth factor receptor messenger RNA are early markers of carcinogenesis in head and neck cancer. Cancer Res 53: 3579-3584, 1993.
37. Vermorken JB, Herbst RS, Leon X, Amellal N and Baselga J: Overview of the efficacy of cetuximab in recurrent and/or metastatic squamous cell carcinoma of the head and neck in patients who previously failed platinum-based therapies. Cancer 112: 2710-2719, 2008. 